\title{
Experiencia clínica
}

\section{Tratamiento anticoagulante oral en pacientes sometidos a remplazo valvular en un hospital general de Antofagasta, Chile}

\author{
Carlos Arce ${ }^{1}$, Ian Mir ${ }^{2}$, Piero Maqueira ${ }^{2}$, Bruno Solari ${ }^{1}$, Javier Labbé $e^{3-4}$. \\ 1 Médico Cirujano; 2 Estudiante de Medicina Universidad de Antofagasta; 3 Cirujano Cardiovascular. \\ 4 Departamento de Cirugía Cardiovascular, Hospital Regional de Antofagasta, Antofagasta, Chile.
}

Introducción: La atención de pacientes con reemplazo valvular cardíaco constituye una actividad importante en cardiología. Si bien el recambio valvular representa una alternativa terapéutica eficaz para el manejo de esta patología, muchos pacientes requieren de tratamiento anticoagulante oral (TACO) para lograr mayor sobrevida.

Objetivos: Describir los resultados terapéuticos obtenidos en un cohorte de pacientes sometidos a recambio valvular, controlados en el Hospital Regional de Antofagasta.

Resultados: Se identificaron 180 pacientes con reemplazo valvular de los cuales 135 cumplieron criterios de inclusión. Hubo 76 mujeres (56,3\%), 59 hombres (43,7\%); la edad global promedio fue 62 años (28-90), 59 años (30-90) en las mujeres y 64 (36-81) en los hombres. La válvula intervenida fue la aórtica en 69 pacientes $(51,1 \%)$, mitral en $60(44,5 \%)$, aór- tica y mitral en $5(3,7 \%)$, y tricúspide en 1 paciente $(0,7 \%)$. No hubo casos de recambio valvular pulmonar. Se instalaron 122 válvulas mecánicas $(90,4 \%)$ y 13 válvulas biológicas $(9,6 \%)$. El INR promedio fue 2,64 (1,11-5,47). Según válvula intervenida el INR promedio fue: mitral 2,50 (1,11-4,89), aórtica: $2,75(1,19-5,47)$, mitral y aórtica: $2,65(1,28-3,74)$, y tricúspide: 1,87 . Del total de cirugías valvulares, 77 $(57,03 \%)$ se encontraron dentro del rango terapéutico deseado: (mitral 33/60, Aórtica 43/69 mitral y aórtica 0/5, Tricúspide: 0/1). 58 pacientes $(42,97 \%)$ se encontraron fuera del rango terapéutico deseado.

Conclusión: Los resultados obtenidos en nuestro centro se encuentran por debajo de las recomendaciones nacionales. La dificultad por obtener mejores resultados refuerza el uso de prótesis biológicas y la implementación óptima de un policlínico de tratamiento anticoagulante (TACO).

\section{Correspondencia:}

Dr. Carlos Arce Nuñez

arce.cnunez@gmail.com 


\section{Oral anticoagulant treatment (OAT) in patients with cardiac valve replacement at a general hospital in Antofagasta, Chile}

Background: Care of patients with cardiac valve replacement often includes the need for anticoagulation which prevents complications that may decrease survival rate.

Aim: to describe the experience with OAT in a cohort of patients with cardiac valve replacement at the Regional Hospital in Antofagasta

Results: 135 out of 180 patients fulfilled inclusion criteria. There were 76 females (56.3\%) and 59 males $(43.7 \%)$, with a mean of 62 years old (28-90), 59 (30-90) in females and 64 (36-81) in males. The valve replaced was the aortic in 69 patients $(51.1 \%)$, the mitral in $60(44.5 \%)$, both the aortic and the mitral valve in $5(3.7 \%)$. Only 1 patients had a tricuspid valve replaced. 122 mechanical valves $(90.4 \%)$ and 13 biological valves $(9.6 \%)$ were implanted. The overall mean INR was 2.64 (1.11 - 5.47). The mean INR value according to the valve replaced was: mitral valve $2.5(1,11-4,89)$, aortic valve $2.75(1.19$ $5.47)$ and mitral plus aortic valve 2.65 (1.28-3.74). Overall, 58 patients were found to be outside the therapeutic target.

Conclusion: These results are less satisfactory than those proposed by national guidelines. The anticoagulant clinic must be optimized and more biological rather than mechanical valves should be used for cardiac valve replacement

Key words: Cardiac surgery, cardiac valve replacement, oral anticoagulation 
Introducción: La patología valvular continúa siendo hoy en día un desafío terapéutico para el cirujano cardiovascular, afectando actualmente a más de cien millones de personas ${ }^{1}$ y realizándose alrededor de 90.000 recambios valvulares al año en Estados Unidos y 50.000 en Europa $^{1-2-3}$. El avance de la tecnología y la depuración de la técnica quirúrgica han permitido disminuir las tasas de morbimortalidad atribuidas a la cirugía de recambio valvular. No obstante, los pacientes sometidos a recambio valvular por prótesis mecánicas continúan siendo un grupo con alto riesgo de padecer complicaciones tromboembólicas, tales como embolia cerebral o trombosis valvulares, incidencia que se reporta alrededor de $0,6-2,3 \%$ y $0,3-1,3 \%$ anual, respectivamente ${ }^{1-2-4}$, y que justifica el uso a largo plazo de TACO como profilaxis tromboembólica ${ }^{2}$.

Los anticoagulantes orales más utilizados en Chile son el Acenocumarol y la Warfarina, antagonistas de la vitamina $\mathrm{K}$ que son monitorizados mediante el INR (International Normalized Ratio) desea dopara cada tipo de paciente, sus factores de riesgo, el tipo de prótesis utilizada (biológica o mecánica), y su ubicación anatómica. Esta última influye considerablemente en el rango terapéutico de INR deseado, ya que existe un mayor índice de tromboembolismo en recambio valvular mitral versus recambio valvular aórtico, con cifras que rodean el 2,0\% y el 4,0\% anual, respectivamente ${ }^{2-4-6}$. Es por ello que hoy en día los valores de INR deseado fluctúan con rangos que van desde INR 2,0-3,0 dependiendo del modelo de válvula aórtica, a INR 2,5-3,5 en los demás tipos de recambio valvular, incluyendo los múltiples ${ }^{2,4}$.

$\mathrm{Si}$ bien existen reportes que muestran beneficios de un manejo anticoagulante oral más agresivo con INR mayor para todos los pacientes $(>3,0)$, el riesgo de complicaciones hemorrágicas aumenta, lo que hace necesario encontrar un equilibrio en el uso del TACO, ya que las complicaciones tromboembólicas y hemorrágicas representan cerca del $75 \%$ de las complicaciones totales en este tipo de pacientes ${ }^{7}$.Todo esto demuestra la importancia de un adecuado control del TACO a fin de prevenir complicaciones en estos pacientes.

El objetivo de este trabajo fue determinar el nivel de cumplimiento del rango terapéutico óptimo deseado durante el manejo con TACO en pacientes sometidos a recambio valvular cardiaco y controlados en el Hospital Regional de Antofagasta.

Materiales y Métodos: Se efectuó un estudio re- trospectivo observacional en pacientes sometidos a recambio valvular cardíaco y controlados en el policlínico de TACO en el Hospital de Antofagasta entre Enero de 1990 y Diciembre de 2014. Los criterios de inclusión fueron: recambio valvular, período entre Enero 1990 y Diciembre 2014 y tener al menos 2 controles en el policlínico de TACO. El criterio de exclusión fue un seguimiento irregular en el policlínico de TACO.

Se midieron variables demográficas y se estimó el valor promedio y la desviación estándar para las variables continuas, considerando como "dentro de rango terapéutico deseado" a todos los pacientes con INR entre 2,5-3,5 para recambio de válvula mitral (RVM), y INR 2,0-3,0 para recambio de válvula aórtica (RVA). En el caso de recambio doble (RVA+RVM), se consideró la anticoagulación en rango de válvula mitral (INR 2,5-3,5). El estudio contó con la aprobación del comité ético científico del Hospital Regional de Antofagasta.

Resultados: De un total de 180 pacientes intervenidos por recambio valvular cardíaco, 135 cumplieron criterios de inclusión. Las principales causas de recambio valvular fueron por estenosis, seguidas por enfermedad reumática e insuficiencia valvular, con un porcentaje menor de pacientes sin registro de causa (Figura 1). Del total de pacientes incluidos, 76 (56,3\%) fueron mujeres y $59(43,7 \%)$ fueron hombres. La edad promedio global fue de 62,1 años $\pm 11,31$ (28-90); la edad promedio para mujeres y para hombres fue 59 años $\pm 11,4$ (39-90) y 64 $\pm 10,38$ (36-81), respectivamente. El tipo de reemplazo valvular (RV) fue: RV Aórtico: 69 (51,1\%), RV Mitral: 60 (44,5\%), RV Aórtico-Mitral: 5 (3,7\%), y RV Tricúspide: $1(0,7 \%)$. No hubo casos de recambio valvular pulmonar (Tabla 1).

Se instalaron 122 válvulas mecánicas $(90,4 \%)$ y 13 válvulas biológicas $(9,6 \%)$. Las válvulas fueron seleccionadas según el médico tratante, las características propias de cada paciente y la disponibilidad de recursos protésicos. El porcentaje de protrombina (\% PT) promedio fue de $37,53 \% \pm 12,67(16,0-84,0)$, con un INR promedio de 2,64 $\pm 0,69(1,11-5,47)$. El INR promedio según válvula intervenida fue: Aórtica: 2,75 $\pm 0,67$ (1,19-5,47), Mitral 2,50 $\pm 0,68(1,11-4,89)$, Aórtica y Mitral: 2,65 \pm $1,01(1,28-3,74)$ y Tricúspide: 1,87 . Del total de cirugías valvulares, $77(57,03 \%)$ pacientes se encontraron dentro del rango terapéutico deseado post-quirúrgico (Aórtica: 43/69, Mitral: 33/60, Aórtica-Mitral: 0/5, Tricúspide: $0 / 1)$, con un total de 58 pacientes $(42,97 \%)$ fuera del rango sugerido (figura 2). 
Tabla 1. Características de los pacientes sometidos a recambio valvular (Promedio, DE, y Porcentaje).

\begin{tabular}{|c|c|c|c|}
\hline Edad (años, б) & 62,1 & $\pm 11,31$ & \\
\hline \multicolumn{4}{|l|}{ Género (n, $\sigma, \%)$ : } \\
\hline - Masculino & 59 & $\pm 10,38$ & $(22,6)$ \\
\hline - Femenino & 76 & $\pm 11,4$ & $(77,4)$ \\
\hline \multicolumn{4}{|c|}{ Intervención quirúrgica (n, \%): } \\
\hline - $\quad$ RVA $^{*}$ & 69 & & $(51,1)$ \\
\hline - $\quad \mathrm{RVM}^{* *}$ & 60 & & $(44,5)$ \\
\hline$\cdot R V A+R V M$ & 5 & & $(3,7)$ \\
\hline - $\quad \mathrm{RVT}^{\star \star \star}$ & 1 & & $(0,7)$ \\
\hline \multicolumn{4}{|l|}{ Prótesis (n, \%): } \\
\hline - Mecánica & 122 & & $(90,4)$ \\
\hline - Biológica & 13 & & $(9,6)$ \\
\hline$\%$ Protrombina $(, \sigma)$ & 37,53 & $\pm 12,67$ & \\
\hline $\operatorname{INR}(, \sigma)$ & 2,64 & $\pm 0,69$ & \\
\hline
\end{tabular}

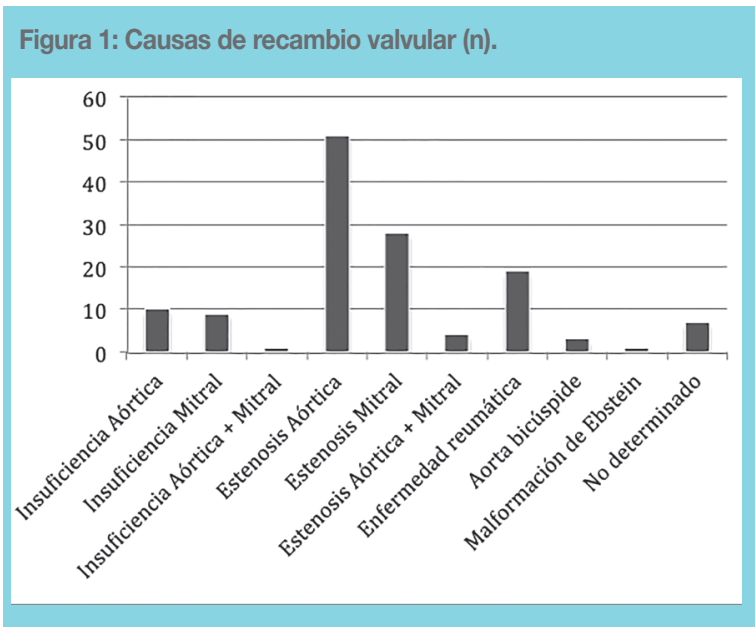

Discusión: Actualmente, la novena edición del "American College of Chest Physicians Evidence-Based Clinical Practice Guidelines" sugiere la utilización de antagonistas de la vitamina $\mathrm{K}$ en todo paciente con prótesis valvular mecánica (evidencia grado 1B), recomendando un INR terapéutico objetivo de 2,5 para RVA (grado 1B), y de 3,0 para RVM o recambio valvular doble (Grado $2 \mathrm{C})^{8-11}$.

En la experiencia que comunicamos aquí, si bien el INR promedio global se mantuvo dentro del rango terapéu-

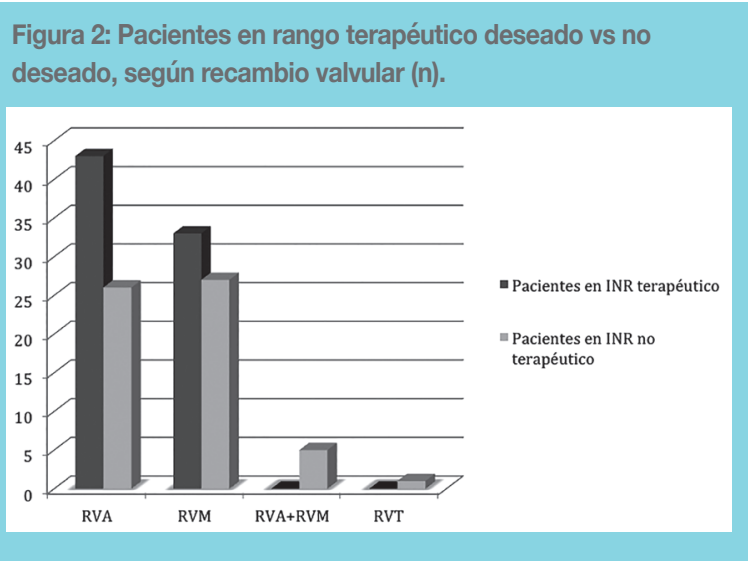

tico deseado, al dividir los objetivos terapéuticos según meta terapéutica individualizada se observa que menos del $60 \%$ de los pacientes $(57,03 \%)$ cumplió con el INR promedio sugerido, valor que se encuentra por debajo de lo recomendado en la literatura nacional $(70 \%)^{12}$. Esto destaca la importancia de un policlínico de TACO que regule y controle los niveles terapéuticos de INR deseados según cada paciente, educando y creando a la vez conciencia respecto a los beneficios y riesgos inherentes al uso de terapia anticoagulante oral ${ }^{9-10-11}$.

Al no disponer de una cohorte más numerosa, no fue posible evaluar los resultados en recambio valvular doble (RVA + RVM) o recambio valvular tricúspide (RVT). Por otra parte, debido a deficiencias en el registro manual que presentan las fichas clínicas de estos pacientes, será necesario complementar estos resultados con nuevos estudios que consideren e incluyan variables tales como el tiempo en rango terapéutico (TTR) y las complicaciones, tanto embolias como hemorragias.

En conclusión, nuestra serie, al comparar los valores de INR promedio de cada paciente, muestra un cumplimiento de rango terapéutico inferior a lo reportado en la literatura, lo cual avala la necesaria implementación de un policlínico de TACO a cargo de profesionales calificados. Paralelamente, los riesgos propios del recambio valvular con prótesis mecánica asociados al importante número de pacientes fuera de rango terapéutico deseado resaltan la importancia de un mayor acceso a prótesis valvulares biológicas. Ello es más atendible dada la mayor duración de las prótesis biológicas y la disponibilidad de implante valvular percutáneo en pacientes con disfunción protésica. 


\section{Referencias}

1. SUN JC, DAVIDSON MJ, LAMY A, EIKELBOOM JW. "Anti- thrombotic management of patients with prosthetic heart val- ves: current evidence and future trends", Lancet 2009; 374 : 565-76.

2. BONOW RO, CARABELLO BA, CHATTERJEE K, DE LEON AC JR, FAXON DP, FREED MD, et al. "ACC/AHA 2006 guidelines for the management of patients with valvular heart disease: a report of American College of Cardiology/American Heart Association Task Force on Practice Guidelines" (Writing Committee to Revise the 1998 Guidelines for the Management of Patients with valvular heart disease): endorsed by the Society of Cardiovascular Anesthesiologists, Society for Cardiovascular Angiography and Interventions, and Society of Thoracic Surgeons. Circulation 2008;118:e523-661.

3. BUTCHART EG, GOHLKE-BÄRWOLF C, ANTUNES MJ, TORNOS P, DE CATERINA R, CORMIER B, et al. "Recommendations for the management of patients after heart valve surgery". Eur Heart J, 2005; 26:2463-71.

4. VINK R, KRAAIJENHAGEN RA, HUTTEN BA, VAN DEN BRINK RB, DE MOL BA, BÜLLER HR, et al. “The Optimal intensity of vitamin $\mathrm{K}$ antagonists in patients with mechanical heart valves: a meta-analysis". J Am Coll Cardiol 2003; 42:2042-8.

5. LEIRIA TL, LOPES RD, WILLIAMS JB, KATZ JN, KALIL RA, ALEXANDER JH. "Antithrombotic therapies in patients with prosthetic heart valves: guidelines translated for the clinician". J ThrombThrombolysis 2011; 31:514-522.
6. SHAN N, DAWSON SL. "Intracerebral haemorrhage, prosthetic valve and anticoagulation”. J R Soc Med, 2004;97:129-30.

7. CHRISTENSEN TD, ANDERSEN NT, ATTERMAN J. "Mechanical heart valve patients can manage oral anticoagulant therapy themselves". Eur J CardiothoracSurg 2003: 23; 292-298.

8. WHITLOCK RP, SUN JC, FREMES SE, RUBENS FD, TEOH $\mathrm{KH}$. "Antithrombotic and thrombolytic therapy for valvular disease: Antithrombotic therapy and prevention of thrombosis", 9th ed: American College of Chest Physicians Evidence-based clinicial practice guidelines. Chest, 2012; 141: e576S-600S.

9. ANSELL JE, BUTTARO ML, THOMAS OV, KNOWLTON $\mathrm{CH}$. "Consensus guidelines for coordinated outpatient oral anticoagulation therapy management". Anticoagulation guidelines task force. Ann Pharmacother 1997; 31: 604-15.

10. BAKER WL, CIOS DA, SANDER SD, COLEMAN CI. Meta-analysis to assess the quality of warfarin control in atrial fibrillation patients in the United States. J Manag Care Pharm 2009; 15: 244-52.

11. MARÍN A, NEIRA V, AIZMAN A. "Eficacia y seguridad del tratamiento anticoagulante oral con antagonistas de vitamina $\mathrm{K}$ en pacientes con prótesis valvulares cardíacas", Rev Chi Cardiol 2014; 33: 27-32.

12. WILSON C, BRAUN S, PARRA C, DUSSAILLANT G, GUARDA E. "Guías para el tratamiento anticoagulante en patología cardiovascular", Comité de educación contínua, Sociedad Chilena de Cardiología y Cirugía Cardiovascular, 2004; 2-10. 